\title{
Gareth R. Eaton: On the Occasion of His 80th Birthday
}

\section{Michael Bowman $^{1} \cdot$ Alistair Fielding $^{2} \cdot$ Mark Tseytlin $^{3}$}

Published online: 27 July 2021

(c) The Author(s), under exclusive licence to Springer-Verlag GmbH Austria, part of Springer Nature 2021

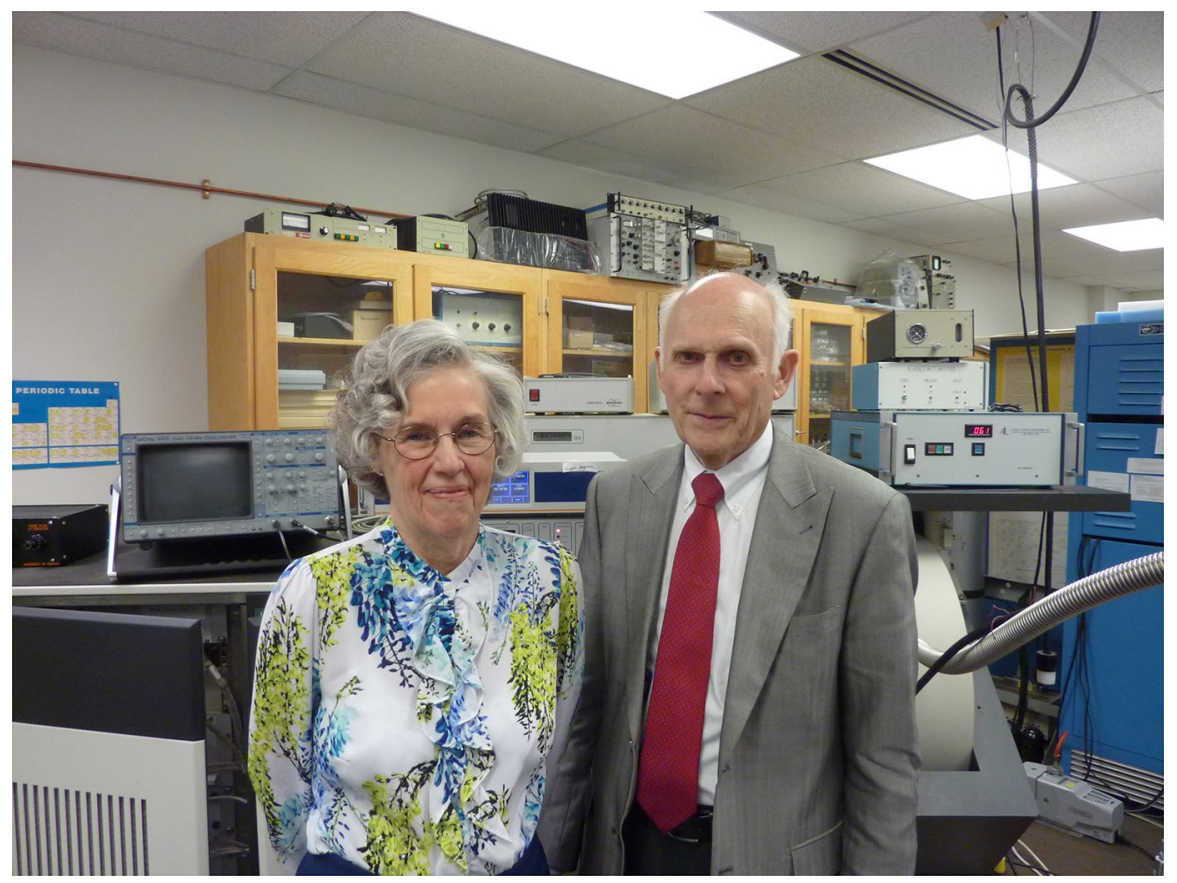

Michael Bowman

mkbowman@ua.edu

Alistair Fielding

A.J.Fielding@1jmu.ac.uk

Mark Tseytlin

mark.tseytlin@hsc.wvu.ed

1 The University of Alabama, Tuscaloosa, Alabama, USA

2 Liverpool John Moores University, Liverpool, UK

3 West Virginia University, Morgantown, West Virginia, USA 
It is with great pleasure that we present this Special Issue of Applied Magnetic Resonance to celebrate the 80th birthday of Prof. Gareth Eaton (John Evans Professorship at the University of Denver, USA) and to recognize the complementary and synergistic role played by Professor Sandra Eaton to what is known as the Denver EPR Group. They have impacted electron magnetic resonance scientifically, technically and socially.

Gareth R. Eaton received his A.B. at Harvard in 1962 and obtained his Ph.D. at MIT in 1972. Since then he has been in the Department of Chemistry (now the Department of Chemistry and Biochemistry) at the University of Denver. He was promoted to Professor in 1980. He served as Dean of Natural Sciences from 1984 to 1988 and as Vice Provost for Research from 1988 to 1989. In 1997 he received the John Evans Professorship at the University of Denver.

Gareth first became interested in magnetic resonance as an undergraduate. Gareth's first book was with William N. Lipscomb on NMR studies of boron hydrides and related compounds, 1969. Early work involved the characterization of inorganic compounds and saw the start of a prolific series of articles on metalnitroxyl interactions characterizing dipolar and exchange interactions with different metal centers and interspin distances in different phases. This period included a seminal paper on the use of the EPR half-field transition to determine interspin distance and orientation. The scientific output of the Denver group touches all aspects of EPR, including: Continuous-wave (CW) and time-domain EPR; structure and dynamics; and spectroscopy, relaxation and imaging. Their studies of spin relaxation times are a reference point for many researchers. They have jointly authored about 380 research papers and book chapters.

Their work also produced major technical innovations in EPR ranging from a sample cell for lossy samples, instrument components developed with RW Quine, including a versatile computer interface for a Varian E9 EPR spectrometer, pulsed EPR spectrometers, and saturation recovery EPR spectrometers. Their developments are incorporated into modern commercial spectrometers, such as EPR signal area measurements, spin quantitation, and rapid scan. The Denver group participated in EPR imaging from its early days, making significant contributions at low frequencies, including resonator design with GA Rinard featuring several innovations such as easily tunable crossed-loop (bimodal) resonators at different frequencies and the use of rapid field scan. This has led to many breakthroughs such as 2D spectral-spatial rapid scan EPR imaging of spin trapped adducts. The rapid scan methodology, including instrumentation and software, has been adopted by several groups worldwide. A commercial rapid scan EPR spectrometer/imager is being developed by the Eaton group in collaboration with Bruker Biospin paving the way for this method to become a mainstream technique similar to pulsed and traditional CW EPR. To this day their output embraces an extremely wide range of EPR applications and the Denver group is a major landmark in the field.

These impressive scientific and technical achievements were accompanied by the nurturing of EPR scientists through the EPR Symposium which they established and ran each summer for over two decades and helped set the stage for the establishment of the International EPR/ESR Society. They brought together the broad EPR community, promoting interactions and communication between the materials, free 
radical and biological branches of EPR. This important conference continues today as the Rocky Mountain Conference. The Eatons have helped to preserve the history of EPR through the book Foundations of Modern EPR written together with Prof. Kev Salikhov which covers personal descriptions of early experiments by pioneers.

Gareth and Sandra S. Eaton jointly received the Bruker Prize in 2002 and became Fellows of the International EPR/ESR Society in 2008.

We hope that this special issue of Applied Magnetic Resonance, containing both original contributions and reviews from friends and colleagues all around the world, will be of great interest to many senior and junior scientists and will provide a glimpse of the different fields touched by the Eaton's in their longstanding career. Fittingly, the contributions range from chemistry-based articles on the characterization of metal complexes and the development of novel spin labels; through biology with applications of hyperfine and dipolar spectroscopy to enzymes; and extending to instrumental development papers on EPR imaging, as well as, papers on the direct dimension in pulse EPR and spin exchange under saturation conditions. The issue features review articles on EPR insights into carotenoids, the current scope of EPR, and a nostalgic retrospective by Professor Harold Schwartz on the influence of the EPR Symposium of the Rocky Mountain Conference.

We heartily thank all the contributors, working against the backdrop of the COVID-19 a pandemic, and we are grateful to Prof. Kev Salikhov and Dr. Laila Mosina for their support and encouragement in all phases of the preparation of this special issue. In much the same way, our thanks go to the referees, who provided constructive criticisms thereby contributing to the improvement of the submitted manuscripts.

Michael Bowman

Alistair Fielding

Mark Tseytlin

Publisher's Note Springer Nature remains neutral with regard to jurisdictional claims in published maps and institutional affiliations. 